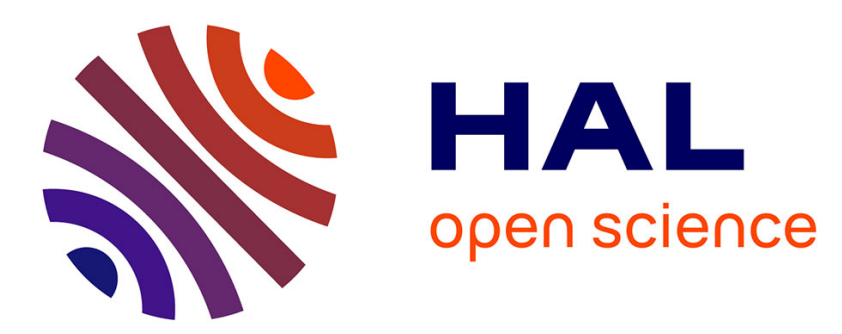

\title{
Acceptabilité des relocalisations des biens face à l'élévation du niveau de la mer: perceptions de nouveaux dispositifs de gouvernance du foncier
}

Helene Rey-Valette, Marie-Laure Lambert, Gisèle Vianey, Bénédicte Rulleau, Camille Andre, Nicole Lautredou-Audouy

\section{To cite this version:}

Helene Rey-Valette, Marie-Laure Lambert, Gisèle Vianey, Bénédicte Rulleau, Camille Andre, et al.. Acceptabilité des relocalisations des biens face à l'élévation du niveau de la mer: perceptions de nouveaux dispositifs de gouvernance du foncier. Géographie, Économie, Société, 2018, 20 (2018/3), pp.359-379. 10.3166/ges.2018.0005 . hal-01950062

\section{HAL Id: hal-01950062 \\ https://hal.umontpellier.fr/hal-01950062}

Submitted on 16 May 2020

HAL is a multi-disciplinary open access archive for the deposit and dissemination of scientific research documents, whether they are published or not. The documents may come from teaching and research institutions in France or abroad, or from public or private research centers.
L'archive ouverte pluridisciplinaire HAL, est destinée au dépôt et à la diffusion de documents scientifiques de niveau recherche, publiés ou non, émanant des établissements d'enseignement et de recherche français ou étrangers, des laboratoires publics ou privés. 


\title{
ACCEPTABILILITE DES RELOCALISATIONS DES BIENS FACE A \\ L'ELEVATION DU NIVEAU DE LA MER : PERCEPTIONS DE NOUVEAUX DISPOSITIFS DE GOUVERNANCE DU FONCIER
}

\section{ACCEPTABILITY OF PROPERTY RELOCATION DUE TO SEA LEVEL RISE: PERCEPTIONS OF NEW MEASURES OF LAND GOVERNANCE}

\author{
Hélène Rey-Valette ${ }^{1 *}$, Marie-Laure Lambert ${ }^{2}$, Gisèle Vianey ${ }^{3}$, \\ Bénédicte Rulleaư ${ }^{4}$, Camille André ${ }^{5,6}$, Nicole Lautrédou-Audouy ${ }^{1}$
}

1. LAMETA, Université de Montpellier, CNRS, Faculté d’Économie, Avenue Raymond Dugrand, CS 79606, 34960 Montpellier cedex 2, France, (helene.rey-valette@umontpellier.fr, Nicole.Lautredou@inserm.fr)

2. LIEU, Université d'Aix-Marseille, 3avRobert Schuman -13100 Aix-en-Provence France (ml.lambert@univamu.fr)

3. Chercheure associée, Université de Tours, 35 allée F. de Lesseps -BP 30553- 37205 Tours Cedex 3 France (gisele.vianey@wanadoo.fr)

4. IRSTEA, UR ETBX, 50 avenue de Verdun, 33612 Cestas cedex, France, (benedicte.rulleau@irstea.fr)

5. EID Méditerranée 165, avenue Paul-Rimbaud 34184 Montpellier Cedex 4 France

6. (maintenant à) GIP Littoral Aquitain, 11, Avenue Pierre Mendès France, 33700 Mérignac, France

*auteur correspondant

RESUME : Face aux risques d'érosion littorale et de submersion marine aggravés par l'élévation du niveau de la mer, de nouvelles mesures, visant à réduire la vulnérabilité des économies littorales par la relocalisation des biens les plus exposés, sont préconisées. Cependant, elles font face à de fortes oppositions, tant des propriétaires concernés que des élus locaux. À partir d'une enquête menée auprès de 421 résidents principaux et secondaires de la commune d'Hyères-les-Palmiers, cet article présente la diversité des perceptions des mesures de relocalisation de façon à analyser les déterminants de leur acceptabilité. Compte tenu de l’importance des contraintes foncières et financières, il étudie notamment la faisabilité de plusieurs procédures de rachat progressif des biens exposés et la capacité de ces dispositifs à renforcer l'acceptabilité des mesures de relocalisation. 
MoTS-CLES : adaptation- changement climatique- gestion du foncier- gouvernance territoriale - relocalisation- risques côtiers-

ABSTRACT: Faced with the menace of coastal erosion and flooding increased by sea level rise, new measures have been put forward that aim to reduce the vulnerability of coastal economies through relocation of property most at risk. These measures have however come up against strong opposition both from land owners and local politicians. From a survey of 421 main and second-home owners in the community of Hyères-les-Palmiers (Var, France), this article presents the range of perceptions of relocation measures in order to analyze the deciding factors in acceptability. Given the importance of land constraints, the article focuses on the feasibility of several schemes for gradually repurchasing property at risk and their ability to strengthen acceptance of relocation measures.

KEYS-WORDS: adaptation, climatic change, land management, territorial governance, relocation, coastal risks.

REMERCIEMENTS : Ce travail a été financé par le programme «Quels littoraux pour demain? » de la Fondation de France dans le cadre du projet «Alternatives Littoral Vulnérable - Élévation du niveau marin (AlternaLIVE)». Les auteurs remercient Sophie Cornet et Alexis Perlade qui ont contribué à l'enquête ainsi que Clotilde Buhot pour sa note bibliographique sur les modalités d’évaluation du foncier.

TITRE COURT : ACCEPTABILILITE DES RELOCALISATIONS DES BIENS FACE A L'ELEVATION DU NIVEAU DE LA MER

\section{ACCEPTABILITY OF PROPERTY RELOCATION DUE TO SEA LEVEL RISE}


L’élévation du niveau de la mer résultant du changement climatique va engendrer une augmentation des dommages lors des tempêtes et des submersions marines devenant plus fréquentes. Dans le cas de la Méditerranée française, un scénario d'un mètre supplémentaire à l'horizon 2100 parait pessimiste mais demeure envisageable compte tenu des prévisions produites par le Groupe Intergouvernemental d'Experts sur le Climat (GIEC, 2014). Or, la densité des constructions est très forte dans les communes littorales concernées et continue de progresser. Entre 1990 et 2012, les nouvelles constructions y ont été trois fois plus nombreuses que la moyenne française (Colas et al., 2015). De plus, ce littoral concentre un tiers des résidences secondaires françaises, lesquelles représentent un quart du nombre total des logements contre $10 \%$ en moyenne dans le pays (Blandy et al., 2016). La région Sud Provence-Alpes-Côtes d'Azur, où se situe Hyères-les-Palmiers, notre terrain d'étude, possède le plus fort pourcentage de terres artificialisées situées à moins de $250 \mathrm{~m}$ des côtes (45 \% contre $23 \%$ en moyenne en France). Au total, en France métropolitaine 140000 habitants vivent à moins de $250 \mathrm{~m}$ de côtes en érosion et 850000 personnes (570 000 logements) habitent dans des zones dites basses, c'est-à-dire à une altitude inférieure à la hauteur atteinte par la mer lors d'une tempête d’occurrence centennale (Colas et al., 2015).

L’importance des populations exposées et la récurrence des dommages qui seront associés à l'élévation du niveau de la mer conduisent donc à préconiser des politiques d'adaptation. Il s’agit de faire évoluer la façon « d’habiter » le littoral (Robert et Melin, 2016). On peut retenir comme définition de l'adaptation celle du GIEC (IPCC, 2001), reprise par la plupart des référentiels et documents institutionnels à savoir : l' « ensemble des actions visant à adapter notre société aux nouvelles conditions environnementales créées par le changement climatique pour en limiter les dommages et maximiser les bénéfices ». Concernant le littoral il s’agit d’assurer la sécurité des personnes ainsi que d’anticiper et réduire les dommages liés aux submersions marines et à l'érosion côtière. Plus généralement il s’agit aussi d’éviter une crise sociale liée à un effondrement du marché immobilier ou aux probables difficultés d’assurance des biens, de réduire les pertes d’aménités et de bien-être pour les populations et de maintenir ou redéfinir l'attractivité des territoires. Aussi les scientifiques et les politiques 
nationales préconisent-ils de nouveaux modes de gestion du trait de côte, privilégiant la relocalisation des biens les plus exposés (MEDDE, 2012). La relocalisation consiste à déconstruire les enjeux (routes ou biens immobiliers) situés en bordure des plages ou des falaises, puis à réaménager de façon douce ces zones (végétalisation, rechargement en sable...) afin qu'elles puissent constituer un espace ou une infrastructure naturelle de protection. Les enjeux peuvent être déplacés ou simplement supprimés. D’abord dénommées «recul stratégique », ces mesures sont à présent qualifiées de « recomposition territoriale » pour insister sur leur intégration dans les projets de territoire. Reste que les études soulignent leur faible acceptabilité, en raison notamment de leur difficile mise en œuvre sur le plan opérationnel, devant le coût qu'elles représentent, l'absence de dispositifs juridiques et financiers dédiés (GIP Littoral Aquitain, 2015), et également l'attachement des individus à leur lieu de résidence et aux aménités littorales (Abel et al., 2011 ; King et al., 2014 ; Huteau, 2015 ; Rey-Valette et al., 2018).

Pour pallier ces contraintes, cet article propose d'étudier les perceptions des habitants littoraux à l'égard de nouvelles modalités de gestion du foncier proposant un rachat progressif des biens exposés. Nous faisons l'hypothèse que ces dispositifs permettraient d’une part de rendre moins douloureux le détachement des populations et donc d'améliorer leur acceptation des relocalisations et d'autre part de réduire les besoins de financement et donc d'accroître leur faisabilité pour les élus (André et al., 2015 ; Gueguen et Renard, 2017). Ces réflexions sont issues d'un projet de recherche pluridisciplinaire sur les coûts et les perceptions des politiques d'adaptation, associant des géomorphologues, des géographes, des économistes et des juristes. Il comparait les arguments et l'acceptabilité de la relocalisation par rapport aux mesures traditionnelles de protection que sont les digues (Rulleau et Rey-Valette, 2017), et étudiait les conditions institutionnelles de mise en œuvre et de gouvernance de la 
relocalisation, en intégrant l'impact de dispositifs juridiques d'indemnisation innovants (Lambert, 2016a).

Les résultats présentés dans cet article se basent sur une enquête menée à Hyères-lesPalmiers, un des sites pilotes du programme d'expérimentation de la relocalisation animé par le Ministère français de l'Écologie, du Développement Durable et de l’Énergie (MEDDE, 2013a-b ; 2015). Cette commune dont le linéaire côtier est important (114km) est confrontée à une forte érosion, notamment (i) sur la presqu'île de Giens formée par un double tombolo créé par les alluvions du Gapeau et de la Maraven et (ii) sur la plaine du Ceinturon où une route fait office de protection sur un littoral très érodé. Du fait de la faible altitude $(1 \mathrm{~m}$ à 1,5 m (MEDDE, 2013a)), une partie de l'habitat pavillonnaire est fortement exposée à la submersion tandis que le front de mer devrait faire l'objet d'une requalification paysagère afin de maintenir sa fonction de protection contre les tempêtes (MEDDE, 2015). Notre réflexion s’intéresse aux modalités de rachat des biens dans une commune qui présente une caractéristique foncière intéressante : un grand nombre de résidents de la zone littorale ne sont pas propriétaires du terrain d'assiette de leur maison, mais titulaires de baux emphytéotiques ${ }^{1}$.

Dans une première partie, nous montrerons en quoi la recomposition des territoires littoraux face à l'élévation du niveau de la mer implique de repenser l’intégration des risques dans les logiques de gestion foncière territoriale. La seconde partie détaillera le protocole méthodologique des enquêtes. Les principaux résultats de l'enquête seront ensuite présentés

\footnotetext{
${ }^{1}$ Le bail emphytéotique qui vient du droit romain, a été formulé pour la première fois en France en 1876 et codifié en 1902 dans le Code rural (arts L451-1 à 13). Il s'agit d'un bail de longue durée (de 18 à 99 ans) qui confère au preneur un droit réel immobilier (Auguet et Carbonnier, 2013). L'emphytéote est l'équivalent du locataire mais il dispose de droits plus importants, notamment celui d'exploiter le bien comme usufruitier, de l'hypothéquer, d'en modifier l'utilisation, et de céder ou transmettre ce droit à ses héritiers. Il paye une redevance généralement faible mais doit assurer des charges d'entretiens et de réparation qui peuvent être importantes. À l'expiration du bail, le bien redevient la propriété du propriétaire-bailleur et l'emphytéote a l'obligation de quitter les lieux sans détruire les aménagements faits et pour lesquels il pourra obtenir une contrepartie. Les baux emphytéotiques peuvent être privés (droit privé) ou administratifs (droit public).
} 
dans la troisième partie puis discutés au regard des modalités d'aménagement et de gouvernance de ces politiques dans une quatrième partie.

\section{Des modes innovants de gestion de la propriété foncière pour faciliter la} relocalisation

La question de l'adaptation au changement climatique s’inscrit dans les problématiques fortement complémentaires de la vulnérabilité et de la résilience des territoires (Gallopin, 2006 ; Miller et al., 2010 ; Turner, 2010) lesquelles mettent en avant le besoin d'adapter les outils d'aménagement. Adopter une logique d'anticipation dans la planification territoriale implique une progressivité des mesures (André et al., 2015) et une temporalité d’inspiration (Levy, 2018) relevant d’une logique de gouvernance d'exploration (March, 1991). Comme l’ont souligné Meschinet de Richemond et Reghezza (2010), la relation entre risque et territoire doit être revue. Ces auteures dénoncent la tendance récente à se focaliser sur les dommages qui conduit à une «déterritorialisation » de la gestion des risques, délaissant l'approche fonctionnelle passée, axée sur la protection des ressources territoriales et le maintien de ses fonctionnalités. Le territoire ne doit en effet pas être entendu comme un simple support du risque (November, 2002) mais, en tant qu'objet socialement constuit (Lupton, 1999), il constitue une composante déterminante des risques qui oblige à intégrer l'histoire, les logiques de gouvernance territoriale (Tiberghein, 2009), les perceptions des populations et les proximités environnementales (Torre et Zuindeau, 2009) et territoriales (Torre et Beuret, 2012). En effet selon Bertrand (2010) l'adaptation au changement climatique doit s’inscrire en continuité de «la mise en territoire du développement durable » impliquant une approche non seulement concertée et intégrée, mais aussi dynamique des projets de territoire dans l'esprit «d'une communauté de destin autour d'éléments géograhiques à fort capital symbolique ». Si l'adaptation aux variations du climat est une pratique ancienne 
(Leroy-Ladurie, 2009), souvent individuelle, l'adaptation au changement climatique implique une approche multidimensionnelle qui ne constitue pas une politique additionnelle mais un nouveau référentiel qui doit faire évoluer l'ensemble des politiques publiques et des pratiques (Urwin et Jordan, 2008 ; Millier et Hubert, 2009). Ainsi, la coordination des décisions privées, souvent sources de mauvaises adaptations (De Perthuis et al., 2009) ou d'iniquités (Godard et al., 2002) est une condition déterminante de l'adaptation et implique une logique de planification prospective (ONERC, 2007). La construction d'un projet d'adaptation s'inscrit alors pleinement dans une problématique d'aménagement du territoire et de régulation de la vulnérabilité territoriale (Kelly et Adger, 2000 ; d’Ercole et Metzger, 2007) qui nécessite des systèmes de gouvernance territoriale adaptés (Adger et al., 2005 ; Duxbury et Dickinson, 2007 ; Abel et al., 2011), afin de renforcer la capacité à s'auto-organiser et à apprendre à s'adapter (Turner, 2010). La régulation de cette vulnérabilité territoriale implique trois composantes : la régulation de l'occupation du sol, des prescriptions pour l'habitat existant et des procédures de gestion des crises (Le Treut, 2018).

La maîtrise de l'occupation du sol suppose une gestion foncière permettant d'articuler les SCOTs et les PLUs avec les plans de prévention des risques littoraux, c’est-à-dire, dans le cas français, d'articuler les logiques du droit de l'environnement, du droit de l'urbanisme et des risques (Le Treut, 2018). Cette articulation est de plus en plus impulsée par des dispositifs tels que les stratégies locales de gestion des risques d'inondation (SLGRI), y compris par submersions marines, mises en œuvre dans le cadre des TRI (Territoires à Risques d'Inondation $^{2}$ ) qui favorisent « l'intégration de la gestion des risques inondation dans toutes les opérations d'aménagement du territoire » (MEDDE 2013-b). La dernière réécriture du code de l'urbanisme (codifiée par l'ordonnance du 23 septembre 2015) précise que «dans le

\footnotetext{
${ }^{2}$ Les Territoires à risque d'inondation (TRI) correspondent aux zones les plus exposées aux inondations au sein d'un bassin versant et pour lesquelles l'État oblige à mettre en place des stratégies locales de gestion de ces risques.
} 
respect des objectifs du développement durable, l'action des collectivités publiques en matière d'urbanisme » vise à atteindre l'objectif de l'adaptation au changement climatique (art L1012-7). Outre la règlementation, le rôle de la fiscalité foncière peut constituer un outil d’orientation et de financement intéressant à mobiliser, par ses capacités à sensibiliser les habitants et à orienter leurs comportements (Guelton, 2016). Selon cette auteure, l'impôt foncier, insuffisamment utilisé, est de plus en plus envisagé comme levier pour réguler les marchés immobiliers, notamment la régulation des terrains non bâtis et la consommation d'espaces naturels. D’autres travaux soulignent aussi le besoin d'outils innovants en matière de financement, d'indemnisation ou d'assurance pour orienter les comportements (Clark, 1998 ; Bagstad et al., 2007 ; Glenk et Fisher, 2010). Enfin, la maîtrise foncière publique, pour la mise en œuvre de programmes d'aménagement, notamment pour la réduction de la vulnérabilité (relocalisation), peut se faire par les outils d’appropriation publique des biens privés: expropriation, préemption ou délaissement. Mais ces outils sont la plupart du temps utilisés sur la totalité du droit de propriété d'un bien, puisqu'en France et contrairement aux pays anglo-saxons, le droit de l'urbanisme et les principes constitutionnels priorisent le droit de propriété individuel. Ainsi malgré l'existence de dispositions pour renforcer l'usage du viager (Drosso, 2007 ; Taffin, 2007) ou du bail emphytéotique pour les transactions les plus chères (Worms, 2011), ces pratiques restent marginales. Peu de travaux portent donc sur le démembrement du droit de propriété dans ses éléments que sont le droit de jouir d’un bien en l'utilisant (usus), d'en percevoir les revenus (fructus) et d'en disposer au sens de le vendre, de le transformer, le donner ou le laisser détruire (abusus). Les distinctions les plus fréquentes établissent une partition entre nue-propriété et usufruit. La dissociation entre usus et fructus, rarement envisagée, se heurte à la difficulté d’évaluer monétairement ces deux composantes. 
Or le démembrement du droit de propriété, qui intervient uniquement lors de transmissions familiales, pourrait pourtant constituer un instrument de politique foncière intéressant pour les collectivités territoriales (Haumont, 2010). Cet outil juridique classique a récemment été repris dans des pistes nouvelles visant la mise en œuvre des relocalisations. Des formes innovantes d'acquisition publique échelonnée des biens vulnérables ont ainsi été proposées par Lambert (2013), Lambert et al. (2011) et André et al. (2015). Elles présentent l'avantage pour les habitants de pouvoir s’accoutumer à l’idée d'abandonner leur logement, et pour les décideurs publics de réduire ou d’échelonner les budgets nécessaires, sachant que ceux-ci seront très importants. En premier lieu, Lambert (2013 et 2016b) propose de définir un «domaine public littoral» permettant au gestionnaire, par préfiguration de l'évolution prévisible du domaine public maritime, de limiter les droits d'usage sur ces zones, voire de les rendre inconstructibles ${ }^{3}$. Lambert (2016b) propose parallèlement un mode d'achat des biens existants échelonné en trois phases : (i) le propriétaire perd immédiatement la nue-propriété (absusus) c'est-à-dire qu'il ne peut plus le vendre et que ses héritiers n'en hériteront pas, mais il peut continuer à le louer et à l'occuper, (ii) au bout de 30 ans, il perd aussi le droit de louer sa propriété (fructus) mais peut toujours continuer à l'occuper et (iii) au bout de 30 ans supplémentaires, il perd finalement le droit de l'occuper (usus).

Dans une logique similaire André et al. (2015) proposent deux modalités de rachat public des biens adaptées aux relocalisations. Il s’agit d’indemniser les propriétaires, soit de la totalité de la valeur du bien en leur laissant un droit d'occupation temporaire leur permettant de rester jusqu'à l'échéance convenue en payant un loyer, soit de la valeur de la nue-propriété uniquement mais en leur permettant de continuer à occuper gratuitement le logement jusqu'à

\footnotetext{
${ }^{3}$ La loi n ${ }^{\circ}$ 86-2 du 3 janvier 1986 relative à l'aménagement, la protection et la mise en valeur du littoral, qui prévoit l'inconstructibilité dans la bande des 100 mètres à partir du rivage (L121-16 Code de l'Urbanisme), permet déjà aux communes d'élargir cette zone inconstructible dans leur plan local d'urbanisme (PLU) « lorsque des motifs liés à la sensibilité des milieux ou à l'érosion des côtes le justifient " (art L121-19 Code de l’Urbanisme), mais uniquement en-dehors des zones déjà urbanisées.
} 
l'échéance de la relocalisation. Ces dispositifs impliquent des frais de gestion non négligeables (André et al., 2015), ainsi que des aménagements pour protéger les biens pendant la période transitoire. Le GIP Littoral Aquitain (2015) évoque enfin la possibilité pour une collectivité territoriale de soumettre l'investissement dans des ouvrages de protection temporaires à l'acquisition de la nue-propriété des biens protégés. Il suggère aussi, pour les biens exposés à plus long terme, d’instaurer un droit de délaissement que pourrait faire jouer les propriétaires à tout moment, mais pour lequel une décote serait appliquée, de plus en plus importante à mesure que l'on se rapproche de l'horizon temporel de la relocalisation. L’ensemble de ces mécanismes garantit une protection transitoire des biens tout en préparant la mise en œuvre effective de la relocalisation.

Ces propositions peuvent être rapprochées du dispositif proposé par Henderson (2018) aux Etats-Unis pour financer et organiser la relocalisation. Cet auteur propose un système dénommé SLPO («Sea Level Purchase Option») qui pourrait être mis en place par les associations fiduciaires foncières chargées de la conservation des écosystèmes. Ces institutions à but non lucratif bénéficient de la confiance des populations, d’un savoir-faire en matière d'achat foncier et de la souplesse de fonctionnement du secteur privé. Il s'agirait de permettre aux propriétaires intéressés de percevoir une rémunération lors de la signature d'une option d'achat et $75 \%$ de la valeur vénale de leur maison à terme lorsque celle-ci sera affectée par la submersion. Une rémunération plus forte pourrait être proposée en contrepartie d’un engagement des propriétaires à ne pas réaliser de protection (digues). Selon l'auteur, ce dispositif volontaire pouvant être mis en place sur une période donnée suffisamment anticipée permettrait de répondre aux principales conditions de faisabilité de la relocalisation, à savoir la flexibilité dans le temps, la réduction des coûts d’achat et la levée des oppositions politiques. 


\section{Méthodologie de l'enquête}

\subsection{Terrain d'étude}

Située en Région Sud - Provence-Alpes-Côte d’Azur, région littorale très touristique, Hyèresles-Palmiers est une commune attractive. Avec 62640 habitants, la croissance démographique d'Hyères-les-Palmiers ( $3^{\text {ème }}$ commune la plus peuplée du département du Var) est liée à son solde migratoire. Elle possède par ailleurs un quart de résidences secondaires (23 \%) et une capacité d’accueil touristique de 11577 lits (Auguet et Carbonnier, 2013). Elle a aussi gardé une activité agricole importante (horticulture, floriculture, pépinières,...), mais contrainte par l'urbanisation. Le fonctionnement hydro-sédimentaire de la plaine côtière a été modifié par plusieurs aménagements portuaires dans les années 1950 et l'érosion des plages en aval des ouvrages a atteint 0,5 m par an en moyenne entre 1972 et 2003 (MEDDE, 2013b).

Cette commune d’Hyères-les-Palmiers a été choisie car elle constitue l’un des cinq sites expérimentaux de relocalisation de l'appel à projets du MEDDE. Lors de l'enquête il est apparu qu'elle permettait aussi d’étudier la situation de nombreux contrats emphytéotiques passés entre la mairie ${ }^{4}$ et des habitants des quartiers de la Capte, la Bergerie, le Pousset et l’Ayguade. Ces baux ont été conclus par la commune d'abord dans les années 1930 puis dans les années 1950: il s'agissait alors de mettre en valeur cette zone marécageuse et de développer le tourisme balnéaire. D’une durée de 70 ans à l'origine, ces baux peuvent être prolongés jusqu'à 99 ans à condition de surélever les constructions d’un étage. Théoriquement, à l'extinction du bail, le bailleur (la commune) récupère la jouissance de son terrain, mais aussi la propriété des constructions réalisées ou des améliorations effectuées. En 1991, une délibération du conseil municipal a cependant autorisé les emphytéotes à racheter

\footnotetext{
${ }^{4}$ Il existe en outre à l'embouchure du fleuve Gapeau, sur la zone dite des Cabanes du Gapeau, quelques baux emphytéotiques alloués par un propriétaire privé
} 
les terrains, permettant par là une sorte de résiliation anticipée du bail. Une clause antispéculative interdisait ce rachat pendant 15 ans après la réalisation du premier étage et permettait à la commune de refuser la vente pour un motif d’intérêt général ou de préservation de ses intérêts. Outre quelques contrats avec des commerces ou des établissements publics, on dénombre actuellement 468 baux emphytéotiques avec des particuliers dont les échéances sont comprises entre 2021 et 2079 (Chambre régionale des comptes, 2017). Bien que les loyers payés soient très sous évalués (de $0,23 €$ à $0,66 €$ le $\mathrm{m}^{2}$, soit des loyers compris entre $16,50 €\left(35 m^{2}\right)$ et $700 €\left(1200 m^{2}\right)$ (Chambre régionale des comptes, 2017)), les conditions de rachat de ces baux et de délai de revente des biens sont des sources de tensions récurrentes entre la municipalité et les emphytéotes ${ }^{5}$. Elles ont été au cœur des débats lors des élections municipales de 2014. L’évaluation effectuée par la Chambre régionale des comptes (2017) témoigne de comportements spéculatifs et de plus-values générées avec des prix de vente des biens qui n’intègrent pas la spécificité des baux ${ }^{6}$, bien qu’ils soient mentionnés dans certaines annonces immobilières. Enfin selon cette évaluation, on note que 38\% des bénéficiaires de ces baux ne résident plus dans la commune (Chambre régionale des comptes, 2017).

\subsection{Conception du questionnaire et protocole d'enquête}

Nous avons distingué trois zones en fonction de l'exposition ${ }^{7}$ à l'aléa de submersion et du fait que les logements soient ou non potentiellement concernés par une politique de relocalisation :

- zone 1 : logements exposés en front de mer donc concernés à la fois par le risque de submersion et potentiellement par la relocalisation ;

\footnotetext{
${ }^{5}$ La recension des articles dans la presse quotidienne régionale entre 2008 et 2014 en témoigne tels que l'article de Nice Matin du vendredi 23 avril 2010 « Baux en suspens : la colère monte sur le littoral » ou celui de Var Matin du 7 juin 2014 « La situation des baux emphytéotiques mobilise le conseil municipal hyérois ».

${ }^{6}$ L'analyse de quelques transactions par la Cour régionale des comptes (2017) témoigne de prix entre $2561 € / \mathrm{m}^{2}$ et $6110 € / \mathrm{m}^{2}$ (voire $11667 € / \mathrm{m}^{2}$ pour un studio) en fonction de la localisation et de la taille.

${ }^{7}$ La définition de la zone d'exposition a été réalisée à partir des cartes du Territoire à Risque d'Inondation (TRI) basée sur l'aléa centennal et intégrant le changement climatique.

http://www.rhone-mediterranee.eaufrance.fr/gestion/inondations/tri.php
} 
- zone 2 : logements situés au-delà de la première ligne donc moins exposés au risque de submersion et non concernés par la relocalisation ;

- zone 3 : logements plus en arrière donc non-exposés au risque de submersion.

Figure 1 : Présentation des zones d’enquêtes

Figure 1 : Presentation of survey areas

Les perceptions des modalités de relocalisation ont été appréhendées en distinguant trois scénarios : (i) un scénario de référence défini en fonction des prévisions des scientifiques du Groupe Intergouvernemental sur l’Évolution du Climat (GIEC, 2014) prévoyant pour 2060 une fréquence des submersions tous les cinq ou six ans, et pour lequel une inaction des pouvoirs publics entrainerait une forte augmentation des cotisations d'assurances, (ii) un scénario de protection par des digues en précisant que celles-ci entraineraient une aggravation de l'érosion des plages et occulteraient la vue sur la mer, (iii) un scénario de relocalisation des infrastructures et des habitations situées en front de mer qui permettrait à la fois d'éviter les dommages, de maintenir les plages, et donc de conserver l'attractivité touristique de la ville.

Le questionnaire comportait 42 questions structurées en cinq rubriques (tableau 1) dont certaines spécifiques pour les habitants en zone 1. L’enquête a été réalisée en trois phases de 15 jours (mai 2014, juillet 2014 et mars 2015) et en face à face au domicile. La passation a mobilisé 4 personnes et chaque questionnaire durait environ $45 \mathrm{mn}$. Au total 421 personnes ont été enquêtées, 14 \% (59) en zone 1, 38 \% (162) en zone 2 et 48 \% (200) en zone 3.

Tableau 1 : Présentation de la structure du questionnaire

Table 1 : Presentation of the questionnaire structure 


\subsection{Caractéristiques des enquêtés}

Notre échantillon est composé majoritairement de résidents à titre principal (88 \%). La part de propriétaires (70\%) est largement supérieure à la moyenne de la commune (49\%). Les répartitions par sexe et par statut familial sont relativement équilibrées et représentatives de la population de la commune (Données communales, INSEE - 2012) avec 54 \% de femmes et 58 \% des couples mariés. Comme à l'accoutumée sur le littoral (Kych, 1986), la part des retraités est importante (45\%). Le niveau de formation est très élevé : 46 \% des enquêtés ont un diplôme correspondant à deux ans d'étude après le baccalauréat, contre 26 \% en moyenne pour la commune. Enfin, 45\% des enquêtés sont adhérents à au moins une association (13\% à une association de protection de l'environnement) et près d'un quart a un lien professionnel avec un des domaines relatifs à notre enquête (tourisme : $14 \%$; milieu maritime : $9 \%$; environnement : $4 \%$ ).

\section{Résultats}

Plus de la moitié des enquêtés (59\%) pensent qu'il est urgent d'envisager le risque d'élévation du niveau de la mer dans les 10 ans à venir tandis que seulement $15 \%$ sont sceptiques, pensant soit que les prévisions ne sont pas prouvées (9 \%), soit que l'élévation du niveau de la mer ne sera pas importante (6 \%). Au quotidien, la plupart est sensible aux alertes météo et en tient compte dans son comportement (48\%); et $10 \%$ se disent même très angoissés dans ces situations. Les enquêtés des zones 1 et 2 déclarent pour près des trois quarts (74 \%) ne pas avoir subi de submersion dans leur logement et ils sont $56 \%$ en zone 1 et $62 \%$ en zone 2 à penser ne pas être exposés à ce risque de submersion, ce qui atteste d'un biais d'optimisme. Concernant la perspective de perte de valeur des biens en cas de submersions répétées, seuls 11 \% des enquêtés des zones 1 et 2 ont estimé que leur bien serait 
quasiment invendable, tandis que selon les enquêtés de la zone 3 cette situation devrait concerner $43 \%$ des biens (Tableau 2).

Tableau 2 : Estimation de la perte de valeur des biens exposés en cas de submersions répétées Table 2 : Estimate of the loss of value for property at risk in the case of repeated flooding

Interrogés quant aux perspectives et au réalisme d'une relocalisation, les enquêtés des trois zones sont seulement $21 \%$ à déclarer que la relocalisation est et sera irréaliste. Ces mesures sont envisagées dans le futur avec $41 \%$ des enquêtés qui estiment que la relocalisation est envisageable dans les vingt ans à venir, et 38\% à plus long terme c'est-à-dire dans cinquante ans ou au-delà. Les habitants de la zone 1 sont proportionnellement plus nombreux à penser que la relocalisation est irréaliste (Test du Chi2 significatif à $1 \%$ ). Il convient à ce propos de signaler le profil spécifique des résidents des zones 1 et 2 qui sont significativement plus propriétaires, plus souvent résidents secondaires (Test du Chi2 significatif à $1 \%$ ) et plus nombreux à avoir plus de 65 ans et à être retraités (Test du Chi2 significatif à $1 \%$ ). Enfin, on observe une différence significative des niveaux de revenu selon la zone, avec un revenu médian de $3750 €$ en zone 1 , contre $2500 €$ en zone 2 et $1650 €$ en zone 3 . Il existe par ailleurs, comme on pouvait s’y attendre, une corrélation entre ces réponses et la perception des risques (Tableau 3). Les personnes qui ne sont pas conscientes des risques (i.e. qui jugent qu’il est inutile de se préoccuper de l'élévation du niveau de la mer car elle ne sera pas importante) ou sont dans le déni (i.e. qui pensent que les prévisions ne sont pas prouvées) ont plus souvent déclaré que la relocalisation est irréaliste (Test du Chi2 significatif à $1 \%$ ). 
Tableau 3 : Avis sur le réalisme des types de mesures d’adaptation

Table 3 : Opinions on the practicality of various types of adaptation measures

Pour les résidents de la zone 1, nous avons aussi cherché à évaluer les effets de l'attachement au lieu et à la mer sur leur qualité de vie à travers une note s’échelonnant sur une échelle de 0 à 10. Les résultats montrent que la perte de la vue sur mer puis la perte des souvenirs sont perçues comme les conséquences de la relocalisation ayant les impacts les plus importants (Tableau 4).

Tableau 4 : Perceptions des effets de la relocalisation par les enquêtés concernés (zone 1)

Table 4 : The affected respondents’ perceptions of the effects of relocation (area 1)

Concernant les intérêts de la relocalisation pour la commune, les enquêtés évoquent principalement le maintien des plages, la mise en sécurité des biens et des personnes et les coûts évités lors des tempêtes futures (Tableau 5). Si ces trois éléments obtiennent des scores relativement voisins en regroupant les choix 1 et 2, les choix effectués en première priorité sont plus contrastés. Ils s’orientent plus vers des aspects récréatifs, environnementaux et sécuritaires (74 \%) que financiers et économiques (26 \%).

Tableau 5 : Intérêts d’une politique de relocalisation pour la commune

Table 5 : Interests of relocation policy for the community

Enfin, notre enquête a évalué l'adhésion des habitants concernés à d'éventuelles modalités innovantes d'achat de leurs biens, ces dernières étant, comme nous l'avons vu, susceptibles de faciliter le détachement par rapport aux biens, donc l'acceptabilité des relocalisations. Il était 
tout d'abord demandé de choisir entre le mode de délaissement de la propriété en trois temps proposé par Lambert (2013 et 2016a) et une expropriation immédiate. Plus de la moitié (53 \%) des enquêtés préfère une perte de propriété échelonnée alors que 27 \% préfèrent être expropriés immédiatement. 20 \% n’ont pas su choisir : cette proportion importante témoigne de difficultés à se projeter dans ce dispositif novateur et inconnu. Une deuxième question permettait d'explorer les autres modalités d'achat proposées par André et al. (2015) et elle révèle que l'autorisation d’occupation temporaire avec un loyer est l'option préférée (46 \%) (Tableau 6).

Tableau 6 : Préférence des options d'achat des logements en cas de relocalisation des biens

Table 6 : Preference for property purchasing options in the case of relocation

Par ailleurs, $80 \%$ des enquêtés de la zone 1 souhaiteraient participer à un processus de concertation s’ils étaient concernés par une opération de relocalisation. Interrogés sur l'éventualité de leur adhésion à une association de propriétaires concernés par la relocalisation et les motifs de cette adhésion, près de la moitié (45 \%) exprime le souhait que la concertation soit accrue (Tableau 7).

Tableau 7 : Volonté et motifs d’adhésion à une association de propriétaires concernés par la relocalisation

Table 7 : Willingness and motives for joining an association of landowners affected by relocation

Concernant les modalités d'indemnisation, $28 \%$ s'expriment en faveur d'un plafonnement des indemnisations (sachant que la valeur des biens en Sud Provence-Alpes-Côte d'Azur peut 
être très élevée), 35 \% pour une minoration de l’indemnisation des résidences secondaires et 62 \% pour que les propriétaires ayant récemment acheté en connaissant le risque soient moins indemnisés. Enfin, les enquêtés sont majoritairement favorables à un financement public national (64\%), régional ou départemental (20\%) de la relocalisation ; seuls $3 \%$ optent pour des taxes locales et $12 \%$ souhaitent que ce type de politique soit financé directement par les bénéficiaires (4 \% par des taxes de séjour et $9 \%$ par des assurances privées).

\section{Discussion}

Globalement les réponses des enquêtés témoignent d’une conscience plutôt partagée de la réalité des risques littoraux sur la commune d'Hyères-les-Palmiers. Cependant un biais d’optimisme, caractéristique des perceptions face au risque (Tversky et Kahneman, 1974 ; Lupton, 1999 ; Gollier, 2004 ; Peretti-Watel, 2005 ; Kouabenan et al., 2007), affecte les réponses des habitants les plus exposés (zones 1 et 2). Il peut être accentué par le fait qu’il s’agit de risques émergents liés au changement climatique (Dutt et Gonzales, 2012). Ainsi, une étude menée en Languedoc-Roussillon a montré que seuls $27 \%$ des résidents littoraux pensent que les submersions sont susceptibles de rendre inhabitables sur le long terme certains quartiers (18 \%) ou l'ensemble de leur commune (9 \%) (Hellequin et al., 2014). L’analyse des profils des enquêtés de la zone 1, les plus concernés, montre qu’ils sont plutôt plus âgés, relativement moins formés, plus souvent propriétaires. Or plusieurs travaux ont montré que ces caractéristiques expliquent un attachement plus fort au bien et une moindre sensibilité au risque de submersion (King et al., 2014 ; Hellequin et al., 2014). De même, on note une proportion plus importante de résidents secondaires en zone 1, lesquels sont généralement plus attachés à leur bien car celui-ci est souvent associé à des souvenirs d’enfance et/ou à des moments familiaux et de convivialité (Hellequin et al., 2014 ; Rey-Valette et al., 2015). 
Notre objectif principal était de tester dans quelle mesure des formes d'appropriation innovantes, permettant un détachement progressif des propriétaires et réduisant les coûts pour les collectivités ${ }^{8}$, pourraient renforcer l'acceptabilité des mesures de relocalisation. L'existence de baux emphytéotiques octroyés par la commune d’Hyères-les-Palmiers aurait pu constituer un atout pour faciliter une politique de réappropriation foncière par la commune. Cependant, il s’avère que les emphytéotes cherchent au contraire à racheter les terrains pour avoir la pleine propriété de leurs biens, ceci générant d’importantes tensions politiques au sujet du prix et des conditions de ces rachats. Pourtant, et malgré les polémiques locales autour de ces baux, nos résultats témoignent d’un intérêt important des habitants pour des mesures innovantes d'achat des biens à relocaliser. Les objectifs étant très différents, les enquêtés n’ont pas rapproché les dispositifs proposés avec les baux en cours sur la commune. En effet, 53 \% des enquêtés sont favorables à une perte progressive des droits (abusus puis fructus puis usus) et 78 \% à une période limitée d’occupation durant laquelle ils deviendraient locataires et auraient à payer ou non une contrepartie selon que l'achat a porté sur la totalité des droits (46\%) ou uniquement sur la nue-propriété (22\%). Soulignons que l'esprit de ces procédures innovantes a été récemment repris dans le cadre des préconisations du Comité National de suivi de la stratégie nationale de gestion intégrée du trait de côte (2015) qui recommande la mise en place de titres d'occupation temporaire. Ces dispositifs progressifs sont cohérents avec le fait que la relocalisation n'est envisagée qu’à moyen terme, sous un délai minimum de 20 ans pour $41 \%$ des enquêtés et de 50 ans et plus pour $38 \%$ d'entre eux. D’après la revue des expériences de relocalisation effectuée par Hino et al. (2017), celles-ci interviennent quasiment toujours après une tempête, comme cela a été le cas en France après la tempête Xynthia. Il est donc difficile de comparer des politiques de relocalisation menées en phase post-crise de réparation, à des projets de territoires menés en anticipation du risque,

\footnotetext{
${ }^{8}$ Cette réduction pouvant atteindre $71 \%$ ou $74 \%$ selon qu'il s'agit d'un dispositif d'achat uniquement de la nuepropriété ou accordant des autorisations d’occupation temporaire assorties de loyers (André et al., 2015)
} 
mettant en œuvre de tels outils d’appropriation. Les procédures d’achat innovantes réduisent les coûts de la relocalisation et renforcent donc la faisabilité financière d'une recomposition territoriale anticipée, mais cette dernière suppose une évaluation de la valeur des biens inférieure à leur valeur vénale actuellement observée alors même que cette dernières n’intègre pas les risques. Ainsi le GIP Littoral Aquitain a testé l'effet d'une variante du mode de calcul des valeurs des biens qui nécessiterait une appropriation publique sur le littoral aquitain, selon plusieurs scénarios de relocalisation à l’horizon 2050. La simulation effectuée sur la base de la valeur dite par capitalisation, basée sur la durée de vie et la valeur locative annuelle, réduirait de moitié les indemnisations par rapport à la valeur vénale (GIP Littoral Aquitain, 2018).

Dans tous les cas cependant, ces procédures innovantes impliquent la mise en place d'une ingénierie adaptée du fait de leur complexité, de leur temporalité et des montants financiers mobilisés (même si elles permettent de réduire ces derniers très sensiblement). De façon à diminuer le recours à l'endettement pour les communes tout en facilitant le portage de ces opérations, il est possible d'envisager qu'elles soient mises en œuvre dans le cadre de partenariats public-privés (Guelton, 2015) avec des promoteurs immobiliers qui seraient alors chargés de leur ingénierie en même temps que des constructions dans les zones de repli (André et al., 2015). Par ailleurs, par rapport à des politiques de laisser faire, la relocalisation anticipée permet de coordonner les anticipations individuelles comme le recommandent Godard et al. (2002) et de mieux garantir la valeur des biens (et donc les intérêts des propriétaires) car ces achats anticipés permettent de maintenir la valeur immobilière prise en compte lors de l'indemnisation, en particulier dans le cas où le bien est acquis en totalité au début de la procédure (André et al., 2015). Cette valeur est intrinsèquement liée au caractère anticipé de la transaction, car le renforcement des dommages au cours du temps impliquera probablement une décote tenant compte du risque avec une baisse progressive ou brutale du 
marché immobilier local. Or l'évaluation de cette décote est aujourd'hui complexe puisqu'elle consiste à évaluer la perte de valeur d'un bien voué à avoir une valeur nulle à l'issue d'une période dont la durée est incertaine. Par exemple, dans le cas particulier des logements insalubres, ce qui pourrait être le cas de logements régulièrement inondés, Melot (2013) rend compte de l'existence dans le cadre de projets d'utilité publique d'une procédure juridique spécifique d'évaluation dite «Méthode de récupération foncière ", et ce lorsque la dégradation du bien le destine à être détruit : le bien concerné est évalué sur la base de la valeur du terrain, après déduction des frais de démolition. Or, la valeur foncière des logements fortement exposés à la submersion est progressivement affectée puisque les terrains seront finalement intégrés dans le Domaine Public Maritime du fait du recul du trait de côte et de l'élévation du niveau marin, alors que c'est l'emplacement du bien qui est le plus valorisé aujourd'hui.

Il est donc nécessaire de définir de nouvelles règles adaptées au changement climatique et de favoriser une approche territoriale facilitant la mise en œuvre de projets intégrés d’adaptation et assurant l'habitabilité des territoires (Richard, 2016). Nous avons à ce propos testé le niveau de connaissance des enquêtés sur le dispositif existant aujourd'hui d'incorporation des biens exposés au recul du trait de côte dans le Domaine Public Maritime sans indemnisation des logements (Code général de la Propriété des Personnes Publiques, articles L. 2111-4 et suivants). Si près de la moitié des enquêtés (48 \%) est informée de ce droit, un tiers estime que l'État n’osera pas l'appliquer à cause de la pression sociale. Depuis ces enquêtes, la jurisprudence a validé ce principe, tout en ouvrant une possibilité de réparation dans des cas limités (Conseil d’Etat 22 septembre 2017, req n400825, concernant la commune de Vias). 
Ces questions d'évaluation seront au cœur des compromis politiques qui devront être réalisés, en amont des relocalisations, sachant que la définition de normes de référence pour les indemnisations est toujours complexe (Melot, 2013). En effet, les enquêtés se sont aussi montrés sensibles à la question de l'équité des modalités d’indemnisation ; ils souhaitent qu’il soit tenu compte de la valeur des biens et de la responsabilité des propriétaires ayant acheté des biens exposés en connaissance de cause. Dans le cadre du financement du rechargement des plages aux Etats-Unis, Mullin et al. (2018) évoquent des pratiques croissantes de taxations locales (taxe foncière) pour pallier la baisse des subventions nationales ou fédérales ; elles sont différentes selon que les propriétaires sont en première ligne et bénéficient directement des externalités des plages ou qu'il s’agit d’habitants plus éloignés des côtes. Selon Mullin et al. (2018), les valeurs peuvent varier de 1 à 10 voire 15 et ces différences ont des conséquences politiques plus ou moins fortes selon la part de résidences secondaires dans la zone et le degré de généralisation de ces pratiques dans les communes voisines. Ainsi, l'accroissement à terme des taxes foncières pourrait aussi être un argument en faveur de l'adhésion à la relocalisation.

Les personnes que nous avons enquêtées souhaitent aussi que des processus de concertation soient mis en œuvre, attestant ainsi de l'importance des conditions de gouvernance, facteur fréquemment évoqué comme contrainte aux relocalisations (Myatt et al., 2003 ; Abel et al., 2011 ; Gibbs et al., 2013 ; Hurlimann et al., 2014 ; Rey-Valette et Rulleau, 2016). Ces attentes confirment la nécessité de penser ces politiques à l'échelle des territoires, en tenant compte également des interactions liées aux différentiels de vulnérabilité et donc en renforçant les solidarités territoriales. Globalement, les perceptions des bénéfices apportés par les relocalisations pour la commune témoignent de motivations environnementales et sécuritaires, avant les aspects financiers ou économiques, ce qui confirme qu'elles sont 
perçues comme relevant de l'intérêt général. En revanche, la hiérarchie des moins-values ressenties par les habitants concernés (zone 1) met l'accent sur des pertes de bien-être individuel surtout liées à la disparition d’aménités et à l'attachement subjectif aux biens. Cet attachement constitue une contrainte majeure comme en témoignent les résistances des populations dans les procédures de relocalisation alors même que le nouveau logement offre des caractéristiques supérieures à celui que les délogés doivent quitter (Faure, 2006). De ce fait, les procédures innovantes de gouvernance foncière proposées constituent, par leur possibilité de détachement progressif, un levier intéressant pour l'adaptation des territoires littoraux. Même si les clauses des contrats devront être très précises et régulièrement rappelées aux propriétaires, acquéreurs ou occupants, on peut imaginer que le climat conflictuel observé dans la gestion des baux à Hyères-les-Palmiers ne se reproduise pas forcément dès lors que le marché immobilier futur sera plutôt orienté à la baisse ${ }^{9}$ et que la multiplication des tempêtes favorisera l'acceptabilité de ces transferts, comme le soulignent de nombreuses enquêtes (King et al., 2014).

\section{Conclusion}

Au final, l'enquête présentée ici montre que, même si la question de la vulnérabilité des biens littoraux reste complexe et sensible, une partie non négligeable des habitants de la commune d'Hyères-les-Palmiers est prête à l'affronter. En revanche, les acteurs publics élus qui devraient porter ces politiques et stratégies émergentes sont moins enclins à mettre en débat ces phénomènes d’aggravation des risques littoraux et leurs solutions potentielles. Ils ont tendance à reporter ces politiques d'adaptation basées sur la réduction de la vulnérabilité, jugées électoralement risquées. Cette tentation politique de remettre à plus tard est contrariée

\footnotetext{
${ }^{9}$ A condition que les doctrines nationales et les politiques locales ne laissent pas entendre que dans tous les cas les habitants seront "protégés » par les collectivités et l'Etat. C'est souvent le cas aujourd'hui avec des investissements de plusieurs millions d'euros dans les digues dans le cadre des PAPI (Programmes d'Actions de Prévention des Inondations) sur les territoires exposés aux submersions marines.
} 
par le droit positif, les intercommunalités ayant à présent acquis la compétence GEMAPI (gestion des milieux aquatiques et prévention des inondations, transférée par la loi du 27 janvier 2014 de modernisation de l'action publique) qui intègre la protection contre les inondations et contre la mer (érosion côtière et submersions marines), mais pour l'instant pas la relocalisation considérée comme une politique de réduction de la vulnérabilité des territoires relevant de l'aménagement du territoire. Cette distinction nuit à une approche globale de l’adaptation par un même porteur. Cependant l’expérience du GIP Littoral aquitain notamment sur la commune de Lacanau, montre qu'à l'issue de multiples actions de sensibilisation et de concertation, si le principe de mesures transitoires de protection avec à terme une relocalisation est de plus en plus discuté et accepté, la décision de mise en œuvre de ces mesures est alors contrainte par les difficultés de financement (Gueguen et Renard, 2017). Plus généralement, on retrouve les constats effectués à l'échelle européenne pour les plans locaux d'adaptation (Aguiara et al., 2018 ; Reckien et al., 2018), quant au rôle déterminant des subventions et des législations avec des situations très différentiées en fonction de la taille des communes. Reckien et al., (2018) mettent en évidence la fonction des programmes de recherche pour initier des approches proactives tandis que dans le même esprit, Birch et Reyes (2018) témoignent de l’influence positive des réseaux collaboratifs et plus généralement de l'existence d'une communauté épistémique en faveur des principes d’adaptation. Enfin, rappelons que ces arbitrages sur les modalités d’indemnisation ou l'assiette fiscale de nouvelles taxes doivent être étudiés en termes d’équité entre populations (Claeys et al, 2017) et à une échelle géographique élargie du fait des transferts d’attractivité susceptibles d'en résulter (Henderson, 2018).

\section{Bibliographie}

Abel N., Gorddard R., Harman B., Leitch A., Langridge J., Ryan A., Heyenga S., 2011. Sea level rise, coastal development and planned retreat: analytical framework, governance principles and an Australian case study, Environmental Science \& Policy, 14, 3, 279-288. 
Adger W.N., Arnell N.W., Tompkins E.L., 2005. Successful adaptation to climate change across scales, Global Environmental Change, 15, 77-86.

Aguiar F.C., Bentz J. Silva J.M.N., Fonseca A.L., Swart R., Duarte Santos F., Penha-Lopes G., 2018. Adaptation to climate change at local level in Europe: An overview. Environmental Science and Policy, 86, 38-63.

André C., Sauboua P., Rey-Valette H., Schauner G., 2015. Acceptabilité et mise en œuvre des politiques de relocalisation face aux risques littoraux : perspectives issues d'une recherche en partenariat, VertigO - la revue électronique en sciences de l'environnement, 15 (1), mis en ligne le 20 mai 2015.

Auguet A., Carbonnier J., 2013. La gestion du foncier littoral par les baux emphytéotiques sur la commune d'Hyères. Mémoire Master 2 Droit de la protection de l'environnement, Territoires Méditerranéens. Univ. Aix Marseille, 68 p.

Bagstad K., Stapletn K., d’Agostino J.R., 2007. Taxes, subsidies and insurance as drivers of United States coastal development. Ecological Economics, 63, 285-298.

Bertrand F., 2010. " Changement climatique et adaptation des territoires », In Zuindeau B. (Ed.), Développement durable et territoire. Lille, Septentrion, 339-350.

Birch T., Reyes E., 2018. Forty years of coastal zone management (1975-2014): Evolving theory, policy and practice as reflected in scientific research publications. Ocean and Coastal Management, 153, 1-11.

Blandy C., Vacher L., Vye D., 2016. Les résidents secondaires, des acteurs essentiels des systèmes touristiques littoraux français ? L'exemple de la Charente Maritime. Territoire en mouvement, Revue de géographie et d'aménagement, 30, mis en ligne le 20 avril 2016.

Chambre Régionale des Comptes - Provence Côte d'Azur, 2017. Rapport d'observations définitives sur la gestion de la commune d'Hyères les Palmiers (Var) à compter de l'exercice 2010, Rapport, 81 p.

Claeys C., Arnaud A., Lambert M.L., 2017, Legal vulnerability worsening environmental inequalities - A case study on coastal populations in Guadeloupe (French Antilles), Comptesrendus Géosciences, 349, 351-358.

Clark M.J., 1998. Flood Insurance as a management strategy for UK Coastal Resilience. The Geographical Journal, 164 (3), 333-343.

Colas S., Duvernoy J., Lasfargues S., 2015. Introduction : le littoral, un espace à forts enjeux, In Le littoral dans le contexte du changement climatique. La Documentation Française, Paris, 11-18.

Comité national de suivi de la stratégie nationale de gestion intégrée du trait de côte, 2015. 40 mesures pour l'adaptation des territoires littoraux au changement climatique et à la gestion intégrée du trait de côte, Rapport MEDDE, 32 p. 
De Perthuis C., 2009. Et pour quelques degrés de plus... Nos choix économiques face au risque climatique. Pearson, Paris.

Drosso F., 2007. Le viager en France : enjeux, blocages et perspectives, Vieillissement de la population et logement. Les stratégies résidentielles et patrimoniales. Bonvalet C., Drosso F., Benguigui F., Huynh P.M., Eds. Sci. La documentation Française, Paris, 463-472.

Dutt V., Gonzales C., 2012. Decisions from experience reduce misconceptions about climate change, Journal of Environmental Psychology, 32, 19-29.

Duxbury J., Dickinson S., 2007. Principles for sustainable governance of the coastal zone in the context of coastal disasters. Ecological Economics, 63, 319-330.

Ercole (d'), R., Metzger, P., 2007. La vulnérabilité territoriale: une nouvelle approche des risques en milieu urbain. Cybergeo: European Journal of Geography, Dossier Vulnérabilités urbaines au sud, document 447, mis en ligne le 31 mars 2009.

Faure S., 2006. De quelques effets sociaux des démolitions d'immeubles. Un grand ensemble HLM à Saint Etienne, Espaces et Sociétés, 124-125, 191-206.

Gallopin G.S., 2006. Linkages between vulnerability, resilience and adaptative capacity. Global Environemental Change, 16 (3), 293-303.

Gibbs M.T., Thébaud O., Lorenz D., 2013. A risk model to describe the behaviours of actors in the houses falling into the sea problem, Ocean \& Coastal Management, 80, 73-79.

GIP Littoral Aquitain, 2018. Sensibilité de l'Aquitaine à l'érosion côtière. Actualisation et nouveaux chiffre clés. Rapport Avril 2018, 70 p.

GIP Littoral Aquitain, 2015. La relocalisation des activités et des biens en Aquitaine Synthèse et conclusion de l'étude de faisabilité de la relocalisation à Lacanau, mai 2015, Rapport GIP Aquitaine, 116p.

Glenk K., Fisher A., 2010. Insurance, prevention or just wait and see? Public preferences for water management strategies in the context of climate change. Ecological Economic, 69, 2279-2291.

Godard O., Henry C., Lagadec P., Michel-Kerjan E., 2002. Traité des nouveaux risques. Gallimard, Coll. Folio Actuel, Paris.

Gollier C., 2004. Les déterminants socio-économiques des comportements face aux risques, Revue économique, 56 (2), 417-422.

Groupe d'experts Intergouvernemental sur l'Evolution du Climat (GIEC), 2014. Cinquième rapport du Groupe d'experts intergouvernemental sur l'évolution du climat. Vol 1, Décembre 2014.

Guéguen, A., Renard, M., 2017. La faisabilité d’une relocalisation des biens et activités face aux risques littoraux à Lacanau, Sciences, Eaux, Territoires, Gestion du risque inondation : connaissances et outils au service de l'aménagement des territoires, 23, 26-31. 
Guelton S., 2016. La fiscalité foncière : des changements de fond. Intercommunalités. AdCF, 3 p.

Guelton S., 2015. La gestion des services publics locaux en partenariat public-privé : une motivation pour assainir les finances locales? Pouvoirs Locaux, les cahiers de la décentralisation, Institut de la décentralisation, Financement de l'action publique : modèles option, I/2015, 16-26.

Haumont F., 2010. Le bail emphytéotique comme outil de contrôle de l'urbanisme. Exemple de Louvain La Neuve, Etudes Foncières, 143, 36-37.

Hellequin A.-P., Flanquart H., Meur-Férec C., Rulleau B., 2014. Perceptions du risque de submersion marine par la population du littoral languedocien : contribution à l'analyse de la vulnérabilité côtière, Nature Sciences Sociétés, 21 (4), 385-399.

Henderson T. R., 2018. Sink or Sell: using estate purchase options to facilitate coastal retreat. Notes, 71, Vanderbilt Law Review, 641-680.

Hino M., Field C.B., Mach K.J., 2017. Managed retreat as a response to natural hazard risk. Nature Climate Change, 7, mis en ligne le 27 mars 2017

Hurlimann A., Barnett J., Fincher R., Osbaldiston N., Mortreux C., Graham S., 2014. Urban planning and sustainable adaptation to sea-level rise, Landscape and Urban Planning, 126, 84-93.

Huteau C., 2015. Gestion des risques littoraux: adaptation ou déplacement de populations ?, In Les risques naturels en zones côtières. Xynthia: enjeux politiques, questionnements juridiques. Laraonde-Clérac C., Mazeaud A. et Michelot A. Eds. Sci. Presses Universitaires de Rennes, 75-89.

IPCC (Intergovernmental Panel on Climate Change), 2001. Coastal zones and marine ecosystems. Climate Change 2001: Impacts, Adaptation, and Vulnerability Chapter 6.

Kelly, P.M., W.N. Adger, 2000, Theory and practice in assessing vulnerability to climate change and facilitating adaptation, Climatic Change, 47, 325-352.

King D., Bird D., Haynes K., Boon H., Cottrell A., Millar J., Okada T., Box P., Keogh D., Thomas M., 2014. Voluntary relocation as an adaptation strategy to extreme weather events. International Journal of Disaster Risk Reduction, 8, 83-90.

Kouabenan D.R., Cadet B., Hermand D., Munoz-Sastre M.T., 2007. Psychologie du risque. Identifier, évaluer, prévenir. De Boeck, coll. Ouvertures Psychologiques, Bruxelles.

Kych A., 1986. L'arrivée des retraités dans les stations du littoral français, Espace, Population, Société, 4 (2), 139-147.

Lambert M.-L., 2016a. Le recul stratégique : de l'anticipation nécessaire aux innovations juridiques, VertigO, la revue électronique en sciences de l'environnement Hors-série 21. [En ligne], 
Lambert M.-L., 2016b. Habiter le littoral en 2040 : quel droit pour un espace vulnérable ? In Habiter le littoral. Enjeux contemporains, Robert S. Ed. Sci., PUP-PUAM, Marseille, 315326.

Lambert M.-L., 2013. GIZC et élévation du niveau marin : vers une gestion innovante des littoraux vulnérables, VertigO, la revue électronique en sciences de l'environnement Horssérie 18 [En ligne],

Lambert M.-L, Affre L., Allouche A., Laffont-Schwob I., Nicolas L., Sabatier F. 2011, "Vulnérabilité des systèmes Littoraux d'une Grande agglomération méditerranéenne VuLiGAM », Rapport final Liteau, 2011, 99 p.

Leroy-Ladurie E., 2009. Histoire humaine et comparée du climat. Tome 3. Le réchauffement de 1860 à nos jours. Fayard, Paris.

Le Treut H., 2018. Anticiper les changements climatiques en Nouvelle-Aquitaine. Pour agir dans les territoires. Rapport Comité Scientifique Régional AcclimaTerra, 490 p.

Levy L., 2018. L'action sur les territoires face au défi d'une temporalité ouverte. L'improvisation comme modèle pour l'action aménagiste? Développement durable et territoires, 9 (2) Le temps des territoires, mis en ligne le 15 juin 2018.

Lupton D., 1999. Risk, Routledge, London and New York.

March, J.G., 1991. Exploration and Exploitation in Organizational Learning. Organization Science, 2 (1), 71-87.

MEDDE (Ministère de l'Écologie, du Développement Durable et de l'Énergie), 2012. Stratégie nationale de gestion intégrée du trait de côte - Vers la relocalisation des activités et des biens, $19 \mathrm{p}$.

MEDDE (Ministère de l'Écologie, du Développement Durable et de l'Énergie), 2013a. Vers la relocalisation des activités et des biens - 5 Territoires en expérimentation - Séminaire national de lancement du 14 février 2013, 35 p.

MEDDE (Ministère de l'Écologie, du Développement Durable et de l’Énergie), 2013b, Plans de Gestion des Risques d'Inondation à l'échelle du district : des TRI aux stratégies locales Premiers éléments de cadrage, $45 \mathrm{p}$.

MEDDE (Ministère de l'Écologie, du Développement Durable et de l’Énergie), 2015. Vers la relocalisation des activités et des biens - 5 Territoires en expérimentation. Actes du séminaire national de Restitution du 30 juin 2015. Paris, 79 p.

Melot R., 2013. La valeur en procès. Expropriation et stratégies judiciaires. ADEF, Paris.

Miller, F., H. Osbahr, E. Boyd, F. Thomalla, S. Bharwani, G. Ziervogel, B. Walker, J. Birkmann, S. Van der Leeuw, J. Rockström, J. Hinkel, T. Downing, C. Folke, and D. Nelson 2010. Resilience and vulnerability: complementary or conflicting concepts ? Ecology and Society, 15 (3), [online]. 
Millier C., Hubert B., 2009. Le changement climatique : s’adapter ou disparaître ? Natures Sciences Sociétés, 17, 1-2,

Mullin M., Smith M.D., McNamara D.E., 2018. Paying to save the beach: effects of local finance decisions on coastal management. Climatic Change,

Myatt L.B., Scrimshaw M.D., Lester J.N., 2003. Public perceptions and attitude towards a forthcoming managed realignment scheme. Freiston Shore, Lincolnshire, UK. Ocean \& Coastal Management, 46, 565-582.

Meschinet de Richemond N., Reghezza M., 2010. La gestion du risque en France : contre ou avec le territoire ? Annales de géographie, 3, (673), 248-267.

November V., 2002. Les territoires du risque, le risque comme objet de réflexion géographique. Edition Scientifiques Européennes, Bern.

ONERC, 2007. Stratégie Nationale d'adaptation au changement climatique. La documentation Française, Paris, 95 p.

Peretti-Watel P., 2005. La culture du risque, ses marqueurs sociaux et ses paradoxes. Une exploration empirique, Revue économique, 56 (2), 371-392.

Reckien D., Salvia M., Heidrich O., Church J.M., Pietrapertosa F, De Gregorio-Hurtado S., D'Alonzo V., Foley A., Simoes S.G., Krkoska Lorencov E.j, Orru H., Orru K., Wejs A. Flacke J., Olazabal M., Geneletti D., Feliu E., Vasilie S., Nador C., Krook-Riekkola A., Matosovic M., Fokaides P.A., Ioannou B.I., Flamos A., Spyridaki N.A., Balzan M.V., Fülop O., Paspaldzhiev I., Grafakos S., Dawson R., 2018. How are cities planning to respond to climate change? Assessment of local climate plans from 885 cities in the EU-28. Journal of Cleaner Production, 191, 207-219.

Rey-Valette H., Robert S., Rulleau B., 2018. Resistance to relocation in flood vulnerable coastal areas: a proposed composite index. Climatic Policy, 1-13,

Rey-Valette H., Rulleau B., 2016. Gouvernance des politiques de relocalisation face au risque de montée du niveau de la mer », Développement durable et territoires, 7 (1), mis en ligne le 11 avril 2016.

Rey-Valette H., Rulleau B., Hellequin A.-P., Meur-Férec C., Flanquart H., 2015. Secondhome owners and sea level rise: the case of the Languedoc-Roussillon region (France), Journal of Policy Research in Tourism, Leisure and Events, 7 (1), 32-47.

Richard E., 2016. L'adaptation aux changements climatiques. Les réponses de l'action publique territoriale. Presses Universitaires de Rennes, Rennes, Coll. Espace et territoires,

Robert S., Melin H., 2016. Habiter le littoral. Entre enjeux de société et enjeux de connaissance. In Habiter le littoral. Enjeux contemporains. Espace et Développement, Presses Universitaires de Provence \& Presses Universitaires d'Aix Marseille, 7-21. 
Rulleau B., Rey-Valette H., 2017. Forward planning to maintain the attractiveness of coastal areas: choosing between seawalls and managed retreat. Environmental Science \& Policy, 72, 12-19.

Taffin C., 2007. Le prêt viager hypothécaire. Vieillissement de la population et logement. Les stratégies résidentielles et patrimoniales. Bonvalet C., Drosso F., Benguigui F., Huynh P.M., Eds. Sci. La documentation Française. PUCA, Paris, 473-484.

Tiberghein B., 2009. La gouvernance des territoires à dangerosité inhérente: stratégies d'adaptation et perspectives d'évolution. Géographie, Economie, Société, 2 (11), 137-160.

Torre A. et Beuret J.E. 2012. Proximités territoriales. Construire la gouvernance des territoires, entre conventions, conflits et concertations, Economica-Anthropos, Paris.

Torre A., Zuindeau B., 2009. Les apports de l'économie de la proximité aux approches environnementales : inventaires et perspectives. Natures, Sciences, Sociétés, 17, 349-360.

Turner B.L., 2010. Vulnerability ad resilience:coalescing or paralleling approaches for sustainability science ? Global Environmental Change, 20, 570-576.

Tversky A., Kahneman D., 1974. The Framing of Decisions and the Psychology of Choice. Science, 211, 453-458.

Urwin K., Jordan A., 2008. Does public policy support or undermine climate change adaptation? Exploring policy interplay across different scales of governance. Global Environmental Change, 18, 180-191.

Worms B., 2011. Le bail emphytéotique : une voie pour aider l'accession dans les zones les plus chères, Anil Habitat actualité, Mai 2011, 1-16. 


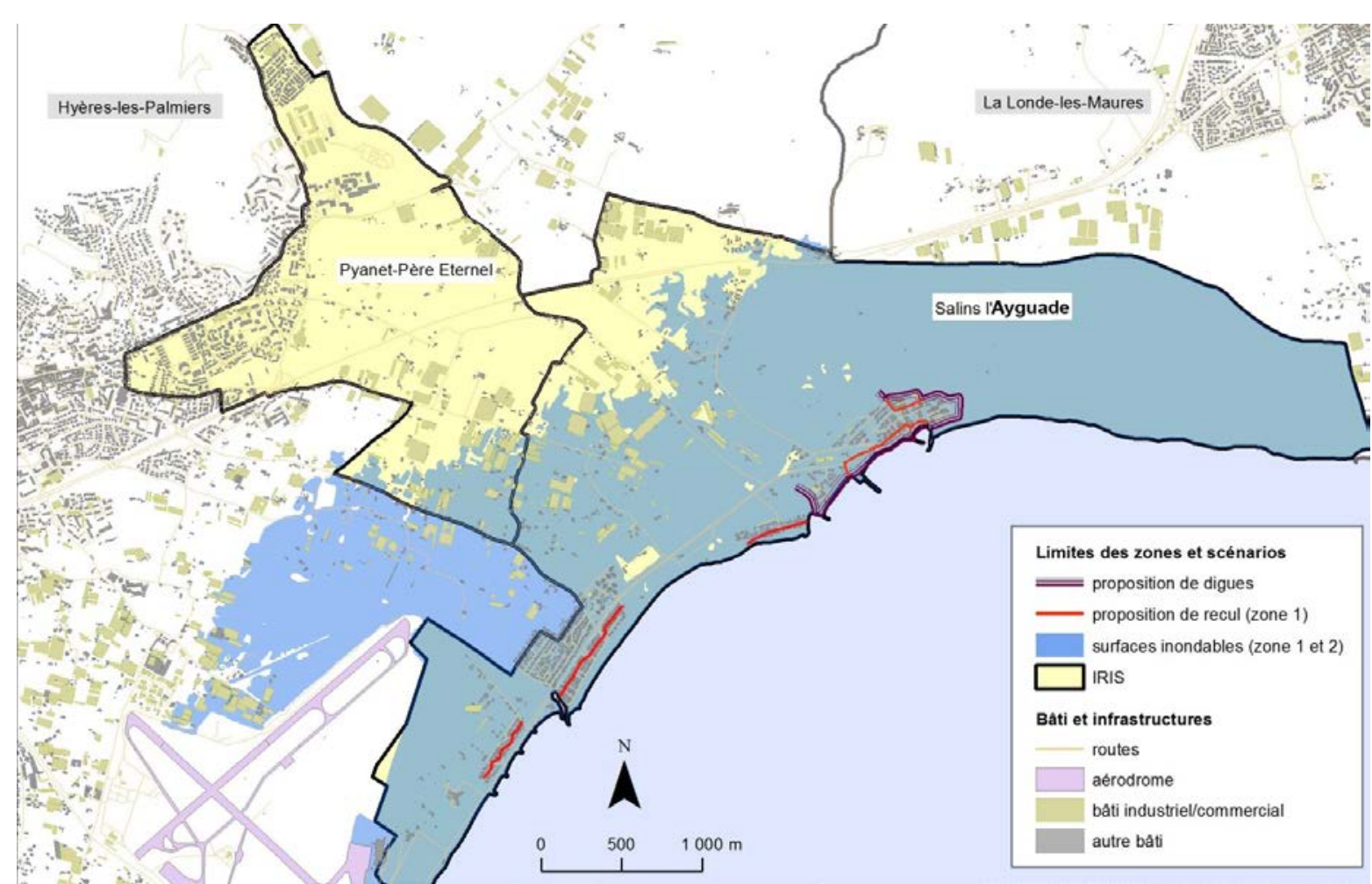

Figure 1 : Présentation des zones d'enquêtes

Figure 1 : Presentation of survey areas

Tableau 1 : Présentation de la structure du questionnaire

Table 1 : Presentation of the questionnaire structure

\begin{tabular}{|l|c|}
\hline Thèmes & Nombre de questions \\
\hline Contexte et attachement au lieu & 7 \\
\hline Perception de la submersion marine & 4 \\
\hline $\begin{array}{l}\text { Position individuelle par rapport aux politiques d'adaptation : } \\
\text { présentation des scénarios et évaluation du consentement à payer pour } \\
\text { une digue et pour une relocalisation }\end{array}$ & 10 \\
\hline Impact de la relocalisation sur la qualité de vie et sur la commune & 4 \\
\hline Perceptions des modalités de relocalisation et principes de justice & 4 \\
\hline Caractéristiques sociodémographiques & 13 \\
\hline
\end{tabular}

Tableau 2 : Estimation de la perte de valeur des biens exposés en cas de submersions répétées Table 2 : Estimate of the loss of value for property at risk in the case of repeated flooding

\begin{tabular}{|l|c|c|}
\hline & $\begin{array}{c}\text { Concernés } \\
\text { (zones 1 et 2) }\end{array}$ & $\begin{array}{c}\text { Non concernés } \\
\text { (zone 3) }\end{array}$ \\
\hline Aucun impact & $46 \%$ & $7 \%$ \\
\hline 10 à $30 \%$ & $22 \%$ & $13 \%$ \\
\hline 30 à $50 \%$ & $19 \%$ & $26 \%$ \\
\hline 50 à $70 \%$ & $2 \%$ & $12 \%$ \\
\hline Les biens seront invendables & $11 \%$ & $43 \%$ \\
\hline
\end{tabular}


Author-produced version of the article published in Géographie, Economie, Société, 2018, 20(3), 359-379

The original publication is available at

https://ges.revuesonline.com/article.jsp?articleld=39835

doi : 10.3166/ges.2018.0005

Source : enquête CEARC LAMETA 2014-2015

Tableau 3 : Avis sur le réalisme des types de mesures d'adaptation Table 3 : Opinions on the practicality of various types of adaptation measures

\begin{tabular}{|l|c|c|}
\hline & $\begin{array}{c}\text { Protection par des } \\
\text { digues }\end{array}$ & $\begin{array}{c}\text { Relocalisation et augmentation } \\
\text { de la taille des plages }\end{array}$ \\
\hline Paraît irréaliste & $39 \%$ & $21 \%$ \\
\hline Paraît réaliste dans 20 ans & $39 \%$ & $41 \%$ \\
\hline Paraît réaliste dans 50 ans & $16 \%$ & $24 \%$ \\
\hline Paraît réaliste plus tard & $6 \%$ & $14 \%$ \\
\hline
\end{tabular}

Source : Enquête CEARC LAMETA 2014-2015

Tableau 4 : Perceptions des effets de la relocalisation par les enquêtés concernés (zone 1) Table 4 : The affected respondents' perceptions of the effects of relocation (area 1)

\begin{tabular}{|l|c|c|c|c|}
\hline $\begin{array}{l}\text { Gradient } \\
\text { d'impact }\end{array}$ & $\begin{array}{c}\text { Séparation des } \\
\text { voisins }\end{array}$ & $\begin{array}{c}\text { Allongement des trajets } \\
\text { (école, médecin...) }\end{array}$ & $\begin{array}{c}\text { Perte de la vue } \\
\text { sur mer }\end{array}$ & $\begin{array}{c}\text { Perte des } \\
\text { souvenirs }\end{array}$ \\
\hline 10 & $32 \%$ & $22 \%$ & $39 \%$ & $36 \%$ \\
\hline 6 à 9 & $7 \%$ & $11 \%$ & $30 \%$ & $25 \%$ \\
\hline 5 & $15 \%$ & $19 \%$ & $12 \%$ & $7 \%$ \\
\hline 1 à 4 & $17 \%$ & $15 \%$ & $12 \%$ & $12 \%$ \\
\hline 0 & $29 \%$ & $32 \%$ & $9 \%$ & $22 \%$ \\
\hline Moyenne & 4,90 & 4,37 & 7,37 & 6,00 \\
\hline
\end{tabular}

Source : enquête CEARC LAMETA 2014-2015

Tableau 5 : Intérêts d'une politique de relocalisation pour la commune Tableau 5 : Interest of relocation policy for the community

\begin{tabular}{|l|c|c|}
\hline & Choix 1 & $\begin{array}{c}\text { Total choix } 1 \\
\text { et } 2\end{array}$ \\
\hline $\begin{array}{l}\text { La plage pourrait être maintenue et agrandie, et le paysage serait plus naturel (motif } \\
\text { récréatif et environnemental) }\end{array}$ & $37 \%$ & $27 \%$ \\
\hline $\begin{array}{l}\text { La commune n'aurait plus de souci de sécurité des personnes en cas de submersion } \\
\text { (motif sécuritaire) }\end{array}$ & $27 \%$ & $25 \%$ \\
\hline $\begin{array}{l}\text { Il n'y aurait plus de dégâts en cas de tempête et cela coûterait moins cher à la société } \\
\text { (motif financier) }\end{array}$ & $19 \%$ & $22 \%$ \\
\hline $\begin{array}{l}\text { Le nouveau quartier construit serait plus moderne et plus écologique (motif } \\
\text { environnemental) }\end{array}$ & $10 \%$ & $12 \%$ \\
\hline L'activité touristique serait maintenue (motif économique) & $7 \%$ & $14 \%$ \\
\hline
\end{tabular}

Source : enquête CEARC LAMETA 2014-2015 
Tableau 6: Préférence des options d'achat des logements en cas de relocalisation des biens Table 6: Preference for property purchasing options in the case of relocation

\begin{tabular}{|l|c|}
\hline $\begin{array}{l}\text { On vous indemnise de la valeur totale de votre logement maintenant et vous pouvez rester } \\
\text { jusqu'à cette échéance avec une autorisation temporaire d’occupation et en payant un loyer }\end{array}$ & $46 \%$ \\
\hline $\begin{array}{l}\text { On vous indemnise de la totalité de la propriété uniquement à l'échéance et vous prenez le } \\
\text { risque que le prix de votre logement ait baissé }\end{array}$ & $32 \%$ \\
\hline $\begin{array}{l}\text { On vous indemnise partiellement (nue-propriété) de votre logement maintenant mais vous } \\
\text { restez gratuitement locataire jusqu'à cette échéance }\end{array}$ & $22 \%$ \\
\hline
\end{tabular}

Source : enquête CEARC LAMETA 2014-2015

Tableau 7 : Volonté et motifs d'adhésion à une association de propriétaires concernés par la relocalisation

Table 7 : Willingness and motives for joining an association of land owners affected by relocation

\begin{tabular}{|c|c|c|}
\hline Adhésion pour avoir une concertation plus importante & $45 \%$ & \multirow{3}{*}{$\begin{array}{l}\text { Adhésion } \\
88 \%\end{array}$} \\
\hline Adhésion pour optimiser l'indemnisation & $19 \%$ & \\
\hline Adhésion pour retarder ou faire échouer le projet de relocalisation & $14 \%$ & \\
\hline Non adhésion car la relocalisation vous semble être la meilleure solution & $20 \%$ & \multirow{2}{*}{$\begin{array}{c}\text { Non adhésion } \\
22 \%\end{array}$} \\
\hline Non adhésion pour être indemnisé avant que le marché immobilier baisse & $2 \%$ & \\
\hline
\end{tabular}

Source : enquête CEARC LAMETA 2014-2015 